\title{
Multilinguales
}

\section{Les doléances en prothèse dentaire : un discours au prisme de la théorie de l'énonciation}

Dolances in dental prosthesis : a speech in the prism of theory of enunciation

\section{Mokhtar Boughanem}

\section{OpenEdition}

\section{Journals}

\section{Édition électronique}

URL : http://journals.openedition.org/multilinguales/4058

DOI : $10.4000 /$ multilinguales.4058

ISSN : 2335-1853

\section{Éditeur}

Université Abderrahmane Mira - Bejaia

\section{Référence électronique}

Mokhtar Boughanem, "Les doléances en prothèse dentaire : un discours au prisme de la théorie de l'énonciation », Multilinguales [En ligne], 11 | 2019, mis en ligne le 17 juillet 2019, consulté le 03 juin 2020. URL : http://journals.openedition.org/multilinguales/4058 ; DOI : https://doi.org/10.4000/ multilinguales.4058

\section{Ce document a été généré automatiquement le 3 juin 2020}

\section{(c) (i) (9)}

Multilinguales est mise à disposition selon les termes de la Licence Creative Commons Attribution Pas d'Utilisation Commerciale - Pas de Modification 4.0 International 


\title{
Les doléances en prothèse dentaire : un discours au prisme de la théorie de l'énonciation
}

Dolances in dental prosthesis : a speech in the prism of theory of enunciation

\author{
Mokhtar Boughanem
}

1 Pour la rédaction de cet article, je me suis appuyé sur ma double formation en médecine dentaire et en sciences du langage. Le sujet développé ici concerne l'analyse discursive des doléances exprimées par les patients partiellement ou totalement édentés ayant bénéficié de traitements prothétiques. Conformément à leur vocation première, les appareillages dentaires constituent une réponse thérapeutique au besoin de rétablir la mastication, la phonation et l'esthétique oro-faciale, des fonctions dont l'altération est causée par la perte des dents naturelles.

2 Etant des «corps étrangers" placés dans la cavité buccale suivant un protocole de conception et de réalisation soigneusement conduit, les prothèses dentaires suscitent parfois des réactions de gêne chez leurs porteurs. Afin de parer à l'éventuel rejet de ces dispositifs, le chirurgien-dentiste programme des séances de contrôle régulières destinées à vérifier leur adaptation.

3 A partir de situations cliniques concrètes, j'envisage d'analyser le mode d'énonciation des doléances verbalisées par les patients. Quelle est la place de la subjectivité dans ce type de productions discursives? Par quelles marques linguistiques se manifeste-telle? A quelle(s) modalité(s) obéissent-ils les énoncés produits dans ce contexte ? Ce sont là les trois principales questions auxquelles je tenterai de répondre dans les lignes qui suivent. 


\section{Doléances post-prothétiques : le point de vue professionnel}

4 Les doléances liées au port des prothèses dentaires font partie de la routine professionnelle des chirurgiens-dentistes. Ces derniers sont suffisamment formés pour les gérer et les prendre en charge en pratique ambulatoire, contribuant ainsi à l'amélioration de la qualité de vie de leur patientèle ${ }^{1}$.

5 De nombreuses publications rendent compte des incidences cliniques de l'inconfort et de l'insatisfaction ressentis par les patients appareillés. L'exercice de la prosthodontie ${ }^{2}$ montre que les prothèses adjointes (amovibles) entrainent, en raison de leur caractère mobile et encombrant, plus de doléances que les prothèses conjointes (fixes). Le patient édenté reste un sujet particulièrement vulnérable, la perte des dents étant souvent assimilée à une mutilation qui laisse des séquelles fonctionnelles, esthétiques, psychologiques et sociales (Lejoyeux, 1980 ; Pompignoli, Raux et Doukhan, 2017).

6 En établissant une typologie des différentes doléances susceptibles d'être rencontrées lors des réhabilitations prothétiques, Adeline Braud, Olivier Hüe et Marie-Violaine Berteretche notent qu'il existe des «doléances objectives ou subjectives, qui peuvent être exprimées de manière immédiate à court terme ou à long terme» $(2007: 1)$. Les doléances dites objectives sont celles qui peuvent être diagnostiquées par le praticien lors d'un examen clinique de routine (instabilité des appareillages, blessures, bruits, etc.). Quant aux doléances subjectives, elles relèvent des sensations éprouvées par le patient à la suite de l'insertion des prothèses. Qu'elles soient objectives ou subjectives, ces doléances peuvent survenir de manière instantanée ou à une date ultérieure.

\section{Pour une approche discursive des doléances post- prothétiques}

7 Ce travail s'inscrit dans le champ de l'analyse du discours, et plus particulièrement dans la théorie de l'énonciation. En tant que cadre de réflexion en situation de déploiement constant, l'analyse du discours s'attache à l'étude des faits de langue à la lumière de leur contexte de production (Maingueneau, 2014). A ce titre, l'énonciation fait partie des nombreux phénomènes pris en charge par l'analyse du discours. Selon Emile Benveniste, celle-ci implique la «mise en fonctionnement de la langue par un acte individuel d'utilisation » (1974: 80). Cette définition renvoie à l'acte langagier par lequel le locuteur appréhende le monde en situation de communication. Partant de ce postulat, les patients appareillés en prosthodontie sont à considérer comme des sujets parlants dès lors qu'ils mobilisent les ressources de la langue dans le but d'exprimer leurs doléances par rapport aux soins prothétiques qui leur sont prodigués. Ces doléances sont le produit d'une activité énonciative plaçant la subjectivité au cœur de tous les enjeux discursifs. En s'adressant à son praticien, le patient nourrit des attentes dont il espère une prise en charge adaptée à sa situation. A ce propos, il convient de préciser que l'absence de travaux antérieurs traitant de ce sujet, en particulier du point de vue discursif, est la principale raison qui m'a motivé à m'y intéresser. Pour ce faire, je me suis appuyé sur la littérature consacrée à la théorie de l'énonciation en général, d'autant plus que celle-ci permet d'appréhender tous types de discours sous l'angle de leur production. 
8 En effet, en dehors des doléances post-prothétiques qui elles aussi peuvent être abordées suivant cette approche, l'étude de la subjectivité dans le langage suscite un vif intérêt auprès d'un nombre considérable de chercheurs. A la suite des travaux d'Emile Benveniste, Catherine Kerbrat-Orecchioni envisage la subjectivité comme étant l'inscription du sujet parlant dans son discours. Sa démarche consiste en «la recherche des procédés linguistiques (shifters, modalisateurs, termes évaluatifs, etc.) par lesquels le locuteur imprime sa marque à l'énoncé, s'inscrit dans le message (implicitement ou explicitement) et se situe par rapport à lui » (1980:32). En effet, la subjectivité est portée par des marqueurs linguistiques, repérables dans le discours, qui témoignent de la présence ponctuelle du locuteur tout au long de ses prises de parole. Selon la terminologie consacrée, ces marqueurs se subdivisent en deux principales catégories : les déictiques et les subjectivèmes (Kerbrat-Orecchioni, 1980). Les déictiques sont des unités linguistiques qui n'ont de sens que dans le cadre d'une situation de communication bien définie. Rentrent dans cette catégorie les pronoms personnels renvoyant à l'émetteur et au récepteur, les démonstratifs et les indices spatiotemporels. Les subjectivèmes, quant à eux, englobent l'ensemble des unités véhiculant un trait affectif ou évaluatif, à l'instar de certains adjectifs, de certains substantifs et de certains verbes et adverbes qui énoncent soit une réaction émotionnelle de la part de l'émetteur, soit un jugement de valeur porté sur un objet quelconque.

9 La position du sujet parlant à l'égard de ce dont il parle renvoie plus spécifiquement au concept de modalisation (Maingueneau, 2002: 382) qui n'est rien d'autre que l'expression de la subjectivité dans et par le discours. Dans la tradition linguistique, il est d'usage de considérer l'énoncé comme une entité à deux constituants : le dictum et le modus (Bally, 1944 [1932]). Alors que le dictum correspond au contenu propositionnel de l'énoncé, le modus réfère à la manière dont le locuteur produit son énoncé. Cette distinction permet d'appréhender le discours comme une construction élaborée par un locuteur dans un contexte donné suivant un objectif qui lui est propre. Le discours est dès lors traversé de modalités qui sont autant de «facettes d'un processus plus général de modalisation, d'affectation de modalités à l'énoncé, par lequel l'énonciateur, dans sa parole même, exprime une attitude à l'égard du destinataire et du contenu de son énoncé » (Maingueneau, 2002 : 384). Dans cette perspective, force est d'admettre, à la suite de Ferdinand Brunot (1953 [1922]) et de Charles Bally (1944 [1932]), que «tout énoncé est modalisé » (Le Querler, 2004 : 645).

10 Un tour d'horizon des différentes modalités décrites dans la littérature (Le Querler, 1996; Meunier, 1974) permet de relever des modalités logiques, volitives, appréciatives et axiologiques. Les modalités logiques requièrent une instance de validation (Gosselin, 2001, 2010) : le réel, le sujet et le système de conventions. En fonction de l'énoncé, elles peuvent véhiculer une valeur aléthique en relation avec le réel, épistémique tributaire de l'opinion exprimée ou déontique liée à l'ordre établi. Les modalités volitives, dites aussi bouliques, traduisent la volonté de l'énonciateur. De leur côté, les modalités appréciatives et axiologiques introduisent des jugements de valeur, charriant ainsi un processus d'évaluation associé soit au caractère désirable/ indésirable d'un procès, soit au caractère louable/blâmable de celui-ci (Gosselin, 2010).

11 Les énoncés soumis à l'analyse dans le présent travail sont recueillis dans le cadre du suivi prothétique des patients appareillés par mes soins. Ma position lors de cette phase de constitution de corpus est avant tout celle d'un praticien soucieux d'évaluer les résultats de mes traitements. Cependant, il convient de signaler qu'en même temps que 
je faisais mon travail de chirurgien-dentiste, je m'attachais à noter avec la plus grande attention qui soit les doléances de mes patients. En règle générale, ces doléances interviennent soit au moment de la livraison de la prothèse, soit lors des séances de contrôle programmées plus tard. Elles sont enregistrées et transcrites suivant l'orthographe usuelle, de manière à faire apparaitre les formes linguistiques employées. Les pauses sont mentionnées par une barre oblique pour faciliter le traitement du corpus au moment de l'analyse.

Dans ce cadre, de nombreuses données contextuelles sont à souligner, étant donné qu'elles participent à la construction du sens au-delà même de ce qui est dit. Ainsi, dans la majorité des cas rencontrés lors de cette étude, qui s'est étalée sur près de deux années d'exercice en tant qu'omnipraticien, les sujets concernés par la réhabilitation prothétique de la cavité buccale ont un âge variant entre 39 et 73 ans. Ceux-ci appartiennent aux deux sexes, masculin et féminin, et consultent pour le motif d'édentement partiel ou total. Dans un pareil contexte, il importe de préciser que «le chirurgien-dentiste est choisi, pas choisissant " (Charon, Joachim, Denys, Auguste et Cottencin, 2014:64), ce qui veut dire que c'est le patient qui choisit son praticien en fonction de ses préférences, et non l'inverse. Indépendamment de son aspect technique, mon rôle consiste dans ce type de situation à laisser mes patients s'exprimer librement dès qu'ils en éprouvent le besoin, notamment lors de l'étape qui consiste à vérifier "le rendu esthétique" au miroir ou au moment de la simulation de la mastication et de l'élocution. Ces derniers ont en effet un profil langagier plurilingue à l'image de la ville d'Alger dans laquelle j'exerce mon métier. Le profil en question se décline en plusieurs catégories de locuteurs : ceux qui s'expriment en arabe algérien, ceux qui s'expriment en kabyle, ceux qui s'expriment en français et ceux qui s'expriment en alternant les codes. Comme mon objet d'étude ici n'est pas d'ordre sociolinguistique, je me suis astreint, pour une meilleure lisibilité de mon corpus, à présenter au cours de mon analyse des énoncés en français. Dans les cas où ces énoncés étaient initialement émis dans une langue autre que le français, la traduction a été faite au mot à mot de sorte à conserver de la manière la plus fidèle qui soit les formes linguistiques sur la base desquelles ont été construits les discours des patients appareillés. Cependant, afin d'éviter la redondance dans cette étude qui se veut d'orientation qualitative, seuls 15 énoncés ont été présentés au cours de l'analyse. Ils ont été sélectionnés en prenant soin de reproduire les marques linguistiques les plus récurrentes qui renvoient à la subjectivité dans l'ensemble du corpus collecté. Ils sont, de ce point de vue, représentatifs de la tendance discursive caractérisant les doléances post-prothétiques.

\section{Les doléances ne sont pas que des doléances}

13 L'expression des doléances en pratique prosthodontique implique l'intervention d'une instance émettrice, le patient appareillé, et d'une instance réceptrice, le praticien. L'enjeu de ces doléances consiste à inviter le praticien à examiner de nouveau ses traitements en vue d'apporter les modifications nécessaires. Cette procédure, somme toute habituelle et naturelle, tient lieu de recours entrant dans le cadre du suivi thérapeutique auquel ont droit les patients. constituent un événement énonciatif résultant de la mobilisation de «l'appareil formel 
de l'énonciation » (Benveniste, 1970). Il s'agit d'une opération par laquelle le patient met en scène, en sa qualité de locuteur, sa vision du monde. Son discours se veut le réceptacle d'indices linguistiques qui révèlent sa présence entre les signes.

Le surgissement du patient en tant que sujet parlant est introduit, dans l'ensemble des situations analysées, par le pronom personnel «je». Celui-ci permet d'établir une relation avec une autre subjectivité, celle du praticien, avec laquelle il est question de communiquer, de dialoguer et d'échanger afin de parvenir à un terrain d'entente. En effet, ce déictique fait référence à un patient dont la réhabilitation prothétique nécessite encore quelques corrections. L'usage de cet élément linguistique sert pardessus tout à actualiser la fonction expressive du langage. En prenant la parole, le patient met en mots une constellation de sentiments qu'il éprouve à la réception de sa prothèse dentaire.

16 Les doléances ne sont donc pas que des doléances. Il s'agit de tout un processus par l'intermédiaire duquel le sujet parlant focalise l'attention de son destinataire sur sa personne propre. L'identité du patient revêt dès lors une dimension sociale, transitoire, valable uniquement en présence du chirurgien-dentiste qui, dans ce cas de figure, endosse une identité professionnelle.

Loin de se présenter comme un bloc homogène, les doléances exprimées par les sujets appareillés entretiennent des valeurs socio-sémantiques aussi diverses que variées. Considérons les énoncés suivants :

docteur / j'ai peur de ne pas pouvoir supporter mon appareil

j'apprécie pas la couleur de ces dents

j'aurais jamais dû me faire remplacer mes dents naturelles

je me sens affreuse avec cette bouche

18 Ces énoncés véhiculent des charges psycho-affectives centrées sur la personne de l'énonciateur. Les doléances dont ils rendent compte s'inscrivent respectivement dans le registre de la crainte, de la désapprobation, du regret et de l'insatisfaction. Bien sûr, il ne s'agit là que d'un aperçu de ce que peuvent être les doléances post-prothétiques.

\section{Les doléances comme appel au secours}

Rien qu'en se prêtant au jeu de l'énonciation, le patient érige le praticien qui se trouve en face de lui en interlocuteur. Les doléances formulées dans ce contexte sont l'expression d'une détresse ressentie à la suite de l'insertion des prothèses dentaires en bouche. La relation qui lie les deux partenaires repose sur un contrat thérapeutique, un contrat à la fois procédural et éthique, conformément auquel le chirurgien-dentiste s'engage à satisfaire du mieux qu'il peut les demandes de ses patients.

20 L'énoncé (1) s'ouvre par la particule " docteur ». Celle-ci se comporte comme un terme d'adresse du moment qu'elle est employée pour interpeller une personne avec laquelle il est question d'établir une communication (Kerbrat-Orecchioni, 1992). Tout en remplissant une fonction relationnelle, la particule «docteur» indique le statut professionnel de la personne à laquelle elle renvoie. Il s'agit d'un titre qui confère à son détenteur la possibilité, voire l'obligation, de porter secours à celui qui en a besoin. Cela fait partie des règles de base qui régissent les métiers de la santé.

21 L'emploi de cette unité sert, au moment de l'énonciation, à attirer l'attention du praticien sur la personne du patient. Ainsi, au-delà de la fonction phatique à travers 
laquelle le locuteur se met en contact avec l'interlocuteur, l'utilisation du terme d'adresse "docteur " montre un patient respectueux des codes sociaux et des usages qu'impose la relation patient-praticien.

\section{«Enlevez-moi ce qabqab que je ne saurais (sup)porter!»}

22 Exerçant dans le contexte algérien, il m'est arrivé à plusieurs reprises d'entendre le mot qabqab [qabqab], qui signifie à l'origine une paire de socques (Lochan, 2017), pour désigner une prothèse peu rétentive ou mal adaptée. Ce mot est spécialement réservé aux prothèses amovibles qui ont la particularité de s'enlever et de se remettre en place à la demande du porteur. Celles-ci ne sont qualifiées comme telles que lorsqu'elles se décollent de façon symptomatique lors de la mastication ou de l'élocution, provoquant de l'inconfort chez l'usager.

L'introduction du mot qabqab dans le paysage linguistique algérien remonte à l'époque ottomane où il n'a été utilisé que pour désigner un type de sabots munis d'une semelle en bois et d'une lanière en cuir. Par extension de sens, il peut servir, le cas échéant, à indiquer tout objet qui provoque du bruit ${ }^{3}$. Dans le cas d'une prothèse dentaire instable ou peu adhérente aux tissus de soutien buccaux, la métaphore semble opérante, surtout si des bruits se font entendre par le patient ou par son entourage lors de la rencontre de la mâchoire inférieure et de la mâchoire supérieure. Cependant, le trait sonore n'est pas le seul paramètre retenu dans ce processus de péjoration dont font l'objet les prothèses à problèmes. Les aspects esthétiques et dimensionnels de ces dernières sont également pris en ligne de compte. Ainsi le mot qabqab désignera-t-il aussi un appareil dentaire grossier qui ne respecte pas les normes de fabrication en vigueur.

\section{Autres manifestations de la subjectivité}

Dans les quatre énoncés présentés plus haut, la subjectivité du locuteur est mise en évidence par des indices d'embrayage et des marqueurs de modalité (Sarfati, 2005). Audelà de l'usage emblématique du pronom personnel «je », précédemment abordé, il existe encore des unités linguistiques qui renvoient à la présence du sujet parlant dans le procès d'énonciation. Par exemple, dans l'énoncé (2), le démonstratif « ces » obéit à un emploi déictique dans la mesure où il place le locuteur dans un rapport d'ostension avec l'objet désigné. Dans ce cas, le référent correspond aux dents artificielles incrustées dans la prothèse exécutée par le praticien. Etant donné que les dents en question se caractérisent par une teinte, une forme et un volume, il se peut que l'une de ces caractéristiques ne réponde pas aux attentes du patient en matière d'esthétique. D'ailleurs, le jugement évaluatif dépréciatif porté sur ces dents se manifeste à travers l'emploi négatif du verbe subjectif « apprécier » conjugué au présent de l'indicatif. Ce procédé traduit une attitude défavorable " de l'agent du procès vis-à-vis de son objet » (Kerbrat-Orecchioni, 1980 : 102). Cette position est reproduite dans l'énoncé (4), mais cette fois-ci avec l'emploi de l'adjectif subjectif « affreuse ». Le sujet parlant est dans ce cas de figure une femme qui n'arrive pas à accepter sa nouvelle situation après la mise en place des prothèses dentaires. 

précédents, il est important de souligner le rôle de certains verbes et adverbes dans l'accentuation de la présence du sujet parlant dans son discours. C'est le cas de l'énoncé (5) où les adverbes "trop » et " beaucoup » expriment l'intensité des procès soumis à l'évaluation par l'énonciateur. A ce niveau, il faut préciser que l'adverbe "trop» renvoie à un procès extérieur à l'énonciateur, à savoir l'épaisseur du bord de la prothèse, tandis que l'adverbe « beaucoup » renvoie à un procès propre à l'énonciateur puisqu'il évalue la gêne ressentie par ce dernier à la suite du port de l'appareillage. Dans la même optique, le verbe "avoir l'impression », employé dans l'énoncé (5), se situe à l'échelle de l'« appréhension perceptive » (Kerbrat-Orecchioni, 1980 : 104), dans la mesure où il permet au locuteur d'engager une relation d'ordre sensoriel avec le monde environnant. De l'autre côté, le verbe "penser ", employé dans l'énoncé (8), relève d'une «appréhension intellectuelle» (Kerbrat-Orecchioni, 1980: 105) de l'expérience évaluative verbalisée par l'énonciateur sous forme d'opinion personnelle. 


\section{De l'énoncé à la modalité d'énoncé}

Les énoncés émis par les patients, dans le cadre de leurs doléances, se voient attribuer différentes modalités. La signification de ces énoncés n'est pas donnée une bonne fois pour toutes, dans la mesure où ils supposent une interprétation qui va au-delà de ce qui est dit. Le choix des procédés linguistiques opéré par le sujet parlant rend visible son attitude et dévoile sa position par rapport à ce qu'il dit et à qui il le dit. L'opacité apparente des énoncés est une caractéristique fondamentale qui justifie leur analyse.

avec un volume pareil ça ne risque pas de tenir

Dans cet énoncé, le patient semble certain de son point de vue. En s'appuyant sur son appréciation subjective du volume de la prothèse, il anticipe le comportement mécanique de cette dernière. En première approximation, son propos donne l'illusion de reposer sur une logique de cause à effet. Or, le volume prothétique est loin d'être le seul critère qui détermine la rétention et la stabilité des prothèses dentaires. Il existe bien d'autres critères qui interviennent dans ce processus, à l'instar des facteurs anatomiques et occlusaux dont il est nécessaire de tenir compte lors de l'examen clinique (Lejoyeux, 1980 ; Pompignoli, Raux et Doukhan, 2017). Cela dit, cet énoncé fait appel à une instance de validation assurée par la subjectivité du locuteur. La modalité qui en résulte est donc de type épistémique, en ce sens qu'elle véhicule la valeur de certitude. Cependant, si l'on veut aborder la situation avec plus d'objectivité, le paradigme épistémique laissera place au paradigme aléthique. Dans l'absolu, l'énoncé en question s'inscrit dans le registre du possible : ce qui est dit peut être vrai comme il peut s'avérer faux. Cet exemple illustre bien le caractère orienté des doléances des patients, leur subjectivité pouvant surgir à n'importe quel moment de l'énonciation. Charge au praticien de faire preuve de prudence face à des situations pareilles, où son travail est mis en cause sur la base de critères foncièrement subjectifs.

En restant dans le cadre des modalités épistémiques, il convient de souligner que nombre d'énoncés analysés mettent en exergue la subjectivité du sujet parlant suivant des procédés plus spécifiques que ceux évoqués plus haut. Les exemples suivants sont destinés à expliciter ce point.

je ne peux pas accepter cet appareil

la prothèse du haut me fait plus mal que celle du bas

je ne crois pas pouvoir un jour mastiquer avec ce dentier

je ne pense pas que mon mari puisse apprécier mon sourire

3 Les énoncés ci-dessus sont tous orientés vers la personne du patient. Dans le premier exemple, il est exclu pour ce dernier de porter la prothèse qui lui a été confectionnée. La possibilité de l'accepter n'est même pas envisageable. Le patient concerné adopte une attitude de rejet à l'égard de son appareillage, un rejet qui peut s'expliquer par une insatisfaction ou un inconfort provoqués par ce dernier.

Derrière leur apparence clairement subjective, les énoncés (12) et (13) affichent une modalité épistémique de probabilité. La douleur et la difficulté de mastiquer sont deux phénomènes auxquels tout patient est exposé après une réhabilitation prothétique de la cavité buccale. Néanmoins, leur intensité de survenue varie d'une situation à l'autre et d'un sujet à l'autre.

Quant à l'énoncé (14), l'accent est mis sur le caractère contestable de la réhabilitation prothétique effectuée. La femme qui prend en charge cet énoncé redoute la réaction de 
dégoût que pourrait éprouver son mari en découvrant les éventuelles insuffisances esthétiques de sa prothèse, notamment au niveau du secteur antérieur. Or, le système d'évaluation des deux partenaires n'est pas forcément identique, à tel point que le mari pourrait même apprécier le résultat du travail.

Parallèlement à ces manifestations modales épistémiques, il existe de nombreux autres énoncés qui n'échappent pas à la modalité appréciative, laquelle insiste sur le caractère désirable ou indésirable du procès (Gosselin, 2010).

je déteste les prothèses à crochets / je ne suis pas prête à avoir un sourire métallique

Dans cet exemple, le verbe «détester ", associé à la personne de l'énonciateur, laisse entendre un jugement dépréciatif relevant de la subjectivité individuelle. Le jugement en question porte sur la nature indésirable des prothèses à crochets. La valeur négative attribuée à ce type de dispositifs est due à leur composition métallique qui, d'après le point de vue du locuteur concerné, affecte le sourire et, partant, toute l'esthétique orofaciale.

38 En guise de conclusion, il convient de rappeler que ce travail aborde les régularités discursives propres aux doléances formulées par les patients pris en charge en dentisterie prothétique. Ces doléances s'inscrivent dans une dynamique énonciative située dans un contexte thérapeutique. Une fois installé sur le fauteuil dentaire, le sujet appareillé se pose comme un locuteur qui se donne les moyens linguistiques et langagiers d'attirer l'attention de son praticien. Les réhabilitations prothétiques sont le sujet de discussion le plus fréquent.

Dans cette perspective, le locuteur s'attache à mettre en avant sa subjectivité à travers un ensemble d'indices linguistiques qui renvoient, directement ou indirectement, à sa personne. Pour étayer ses jugements, il se réfère à une échelle d'évaluation qui repose sur des valeurs d'ordre technique, esthétique, relationnel et social. Les énoncés ainsi produits sont nécessairement colorés, orientés et marqués par des émotions, des impressions et des croyances verbalisées.

La subjectivité constitue donc l'instance de validation la plus privilégiée dans les doléances post-prothétiques. En accompagnant le discours des patients, la modalité épistémique et la modalité appréciative correspondent au lieu de réalisation de cette subjectivité. «La modalité a son origine dans la subjectivité fondamentale de la mise en acte de la langue dans la parole » (Curea, 2015 : 166). De ce fait, l'activité énonciative du locuteur reste inséparable du surgissement de sa personne. Le contrôle de l'adaptation des réhabilitations prothétiques en dentisterie n'est en cela qu'un contexte, voire un prétexte, pour l'expression d'une subjectivité légitimée par la nature de la relation patient-praticien, basée sur des présupposés éthiques qui rendent indispensable le suivi thérapeutique. 


\section{BIBLIOGRAPHIE}

AKIN, S., « La réduplication en kurde kurmanji : aspects morphologiques et sémantiques », in Repères DoRiF, $\mathrm{n}^{\circ}$ 13, 2017. Disponible sur [http://dorif.it/ezine/ezine_articles.php?id=350] (consulté le 09/10/2018)

BALLY, Ch., Linguistique générale et linguistique française, A. Francke, Berne, 1944 [1932].

BENVENISTE E., « L'appareil formel de l'énonciation », in Langages, n 17, 1970, pp. 12-18.

BENVENISTE, E., Problèmes de linguistique générale II, Gallimard, Paris, 1974.

BRAUD, A., HÜE, O., BERTERETCHE, M.-V., « Doléances en prothèse complète », in EMC, (odontologie, 23-325-G-15), Elsevier Masson SAS, Paris, 2007, pp. 1-8.

BRUNOT, F., La pensée et la langue, Masson Paris, 1953 [1922].

CHARON J., JOACHIM F., DENYS K., AUGUSTE N., COTTENCIN O., Relation humaine et communication au cabinet dentaire. Aspects particuliers en parodontie, Editions CdP, Paris, 2014

CUREA, A., Entre expression et expressivité : l'école linguistique de Genève de 1900 à 1940. Charles Bally, Albert Sechehaye, Henri Frei, ENE Editions, Lyon, 2015.

GOSSELIN, L., « Le statut du temps et de l'aspect dans la structure modale de l'énoncé. Esquisse d'un modèle global », in Syntaxe et sémantique, $n^{\circ} 2,2001$, pp. 57-80.

GOSSELIN, L., Les modalités en français. La validation des représentations, Rodopi B.V., AmsterdamNew York, 2010.

GRIGORE, G., L'arabe parlé à Mardin. Monographie d'un parler arabe " périphérique », Editura UniversităȚi din București, București, 2007.

KERBRAT-ORECCHIONI, C., L'énonciation. De la subjectivité dans le langage, Armand Colin, Paris, 1980.

KERBRAT-ORECCHIONI, C., Les interactions verbales 2, Armand Colin, Paris, 1992.

LEJOYEUX, J., Traitement de l'édentation partielle et totale, Maloine, Paris, 1980.

LE QUERLER, N., Typologie des modalités, PUC, Caen, 1996.

LE QUERLER, N., " Les modalités en français », in Revue belge de philologie et d'histoire, $\mathrm{n}^{\circ}$ 82, 2004, pp. 643-656.

LOCHAN, C., « Eléments d'histoire de l'enseignement de la langue arabe en France », dans SAKER, H. (coord.), L'arabe langue de culture universelle, Maury S.A.S., Paris, 2017, pp. 85-92.

MAINGUENAU, D., « Modalisation », dans CHARAUDEAU, P., MAINGUENEAU, D. (dir.), Dictionnaire d'analyse du discours, Editions du Seuil, Paris, 2002, pp. 382-383.

MAINGUENAU, D., « Modalité », dans CHARAUDEAU, P., MAINGUENEAU, D. (dir.), Dictionnaires d'analyse du discours, Editions du Seuil, Paris, 2002, pp. 383-386.

MAINGUENEAU, D., Discours et analyse du discours. Introduction, Armand Colin, Paris, 2014.

MEUNIER, A, « Modalités et communication », in Langue française, n 21, 1974, pp. 8-25.

POMPIGNOLI, L., RAUX, D., DOUKHAN, J.-Y., Prothèse complète. Clinique et laboratoire, Editions CdP, Paris, 2017. 
SARFATI, G.-E., Eléments d'analyse du discours, Armand Colin, Paris, 2005.

SAUSSEY, E., Les mots turcs dans le dialecte arabe de Damas, Imprimerie catholique, Beyrouth, 1929.

\section{NOTES}

1. L'ensemble des patients.

2. La prosthodontie est le nom de la spécialité dentaire qui s'occupe de la réhabilitation prothétique de la cavité buccale.

3. En réalité, le mot qabqab est «d'origine onomatopéique » attestée en arabe (Grigore, 2007 : 149), en turc (Saussey, 1929), en kurde (Akin, 2017) et dans bien d'autres langues.

\section{RÉSUMÉS}

Le paradigme énonciatif offre un éclairage théorique pertinent pour l'appréhension de la subjectivité dans le discours. Le passage de la langue au discours implique l'intervention d'un locuteur dont le rôle consiste à mobiliser «l'appareil formel de l'énonciation». Le positionnement du locuteur se manifeste dès lors à travers des indices linguistiques qui forment un système de repérage déictique et modal.

Partant de ce cadrage théorique, cet article se propose de traiter du fonctionnement énonciatif des doléances consécutives au port des prothèses dentaires. Le sujet appareillé se pose comme un locuteur pour lequel la langue sert à exprimer son sentiment vis-à-vis des traitements qui lui sont réalisés. L'analyse proposée dans ce cadre se veut d'orientation qualitative dans la mesure où elle vise à dégager les régularités énonciatives inhérentes aux doléances post-prothétiques.

The enunciative paradigm offers a relevant theoretical perspective for the apprehension of subjectivity in discourse. The passing from language to speech implies the intervention of a speaker whose role is to mobilize "the formal apparatus of enunciation". The positioning of the speaker is then manifested through linguistic indicators that form a deictic and modal tracking system.

Starting from this theoretical framework, this article proposes to deal with the enunciative functioning of the complaints following the wearing of dental prostheses. The subject wearing dental prothesis stands as a speaker for whom the language serves to express his feeling towards the treatments that have been made to him. The analysis proposed in this context is intended to be qualitative in that it aims to identify the enunciative regularities inherent to post-prosthetic complaints.

\section{INDEX}

Mots-clés : doléances post-prothétiques, énonciation, jugement évaluatif, modalité, subjectivité Keywords : evaluative judgment, modality, post-prosthetic complaints, enunciation, subjectivity 
AUTEUR

MOKHTAR BOUGHANEM

Universitéd'Alger 2, Algérie 